\title{
128-Bit Multicomparator
}

\section{CARVER A. MEAD, RICHARD D. PASHLEY, MEMBER, IEEE, LEE D. BRITTON, YOSHIAKI T. DAIMON,} AND STEWART F. SANDO, JR., MEMBER, IEEE

\begin{abstract}
A 128-bit multicomparator was designed to perform the search-sort function on arbitrary length data strings. Devices can be cascaded for longer block lengths or paralleled for bit-parallel, wordserial applications. The circuit utilizes a 3-phase static-dynamic shift register cell for data handling and a unique gated EXCLUSIVE-NOR circuit to accomplish the compare function. The compare operation is performed bit parallel between a "data" register and a "key" register with a third "mask" register containing DON'T CARE bits that disable the comparator. The multicomparator was fabricated using $\mathrm{p}$-channel silicongate metal-oxide-semiconductor (MOS) technology on a $107 \times 150$ mil chip containing 3350 devices. With transistor-transistor logic (TTL) input, data rates in excess of $2 \mathrm{MHz}$ have been attained. The average power dissipation was $250 \mathrm{~mW}$ in the dynamic mode and 300 $\mathrm{mW}$ in the static mode.
\end{abstract}

\section{INTRODUCTION}

$\mathrm{O}$ VER the past several years, there have been significant amounts of energy devoted to the fabrication of larger and faster semiconductor memories and conventional central processing units (CPU's) in chip form. In the process, many other applications of large-scale integration (LSI) to computer architecture have been neglected [1]. LSI has removed the technological distinction between logic and memory. It is now economically feasible to decentralize the CPU of a computer by replacing much of its maintenance software with functional hardware to improve system efficiency. Presently, an inordinate amount of processing time is spent on organizing and accessing files in peripherals. Peripherals are usually controlled directly by the CPU and have little or no associated logic of their own. A great improvement in this situation can be made by developing peripheral logic units. This would allow each peripheral to accomplish its own internal processing and thus reduce CPU housekeeping duties. This paper describes a 128-bit multicomparator that is designed to perform the search-sort function.

The block diagram of the multicomparator is shown in Fig. 1. The circuit consists of three independently clocked staticdynamic shift registers with associated EXCLUSIVE-NOR gating. In operation, the device indicates a match between the data word and the unmasked bits of the key word. The multicomparator is loaded with a key word by serially shifting the word into the key register and locking the register in static mode. While the key word is being loaded, the comparator is enabled by entering zeros ${ }^{1}$ in the appropriate locations of the

Manuscript received March 15, 1976; revised July 18, 1976.

C. A. Mead is with the California Institute of Technology, Pasadena, CA 91125 .

R. D. Pashley and S. F. Sando, Jr., are with the Intel Corporation, Santa Clara, CA

L. D. Britton is with the Hewlett-Packard Laboratories, Cupertino, CA.

Y.T. Daimon is with the Sony Corporation, Tokyo, Japan.

"Voltage convention: high-" $1 "-V_{D D}$, low-" 0 "- $V_{C C}$. Note that since $V_{D D}$ is negative for $\mathrm{p}$-channel MOS and positive for n-MOS transistor-transistor logic (TTL) levels may or may not have reverse polarity depending on the processing used.

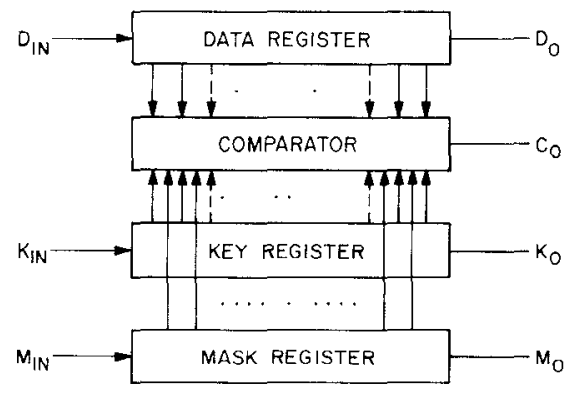

Fig. 1. Block diagram of multicomparator.

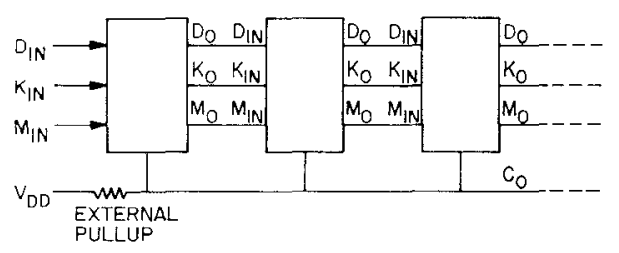

(a)

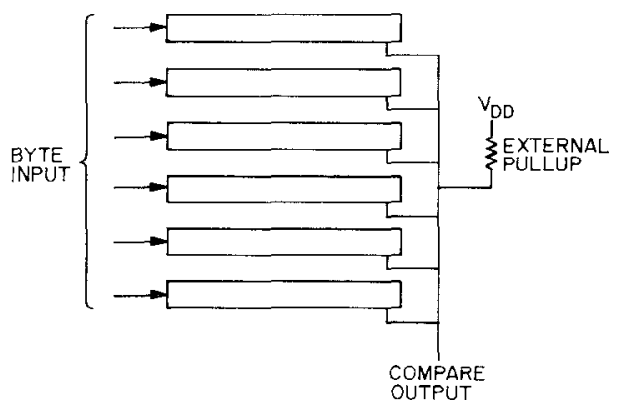

(b)

Fig. 2. Possible connections of multicomparator. (a) Cascaded. (b) Bit-parallel, word-serial.

mask register. Masking allows the multicomparator to search for bit strings of varying length and composition. For example, assume it is necessary to search for all words containing a specific 113-bit code. By entering the 113-bit code in the key register and masking out the rest of the comparator, the multicomparator is conformed to search for this code wherever it occurs in the data file. Once the multicomparator is loaded with "key" and "mask" words, the file being searched is serially shifted through the data register. The data words are compared in bit parallel with the unmasked bits of the key word as they pass through the data register. When a match is found, the compare output goes high.

Large multicomparators can be constructed of the 128-bit circuits. Cascaded [Fig. 2(a)], the comparator can be used to search for words longer than 128 bits. By implementing multicomparators in parallel [Fig. 2(b)], a word-serial, bit-parallel 
organization is achieved which is completely compatible with byte oriented machines. For example, an arbitrary length string of ASCII characters (such as one line of a source listing) can be easily searched using this technique, making the device ideal for indexing, test, etc.

There are many applications for the multicomparator in machine construction. As a result of its serial-search nature, the multicomparator is ideally suited to interface serial-access devices such as tapes or disks. For example, a "smart disk" can be constructed which has as a part of its instruction set, "find the following string." The specification of the string of course would allow DON'T CAREs to mask out certain fields. For example, bin sorting could be accomplished in this manner. This capability coupled with a standard microprocessor would provide a disk capable of doing a large fraction of the string manipulation now done by CPU's. Such string manipulation capability on the word level would be useful in text processing applications. Many of the Snobol-type instructions can be directly implemented using the search chip as input to the processor.

\section{Chip Design}

The multicomparator was fabricated using $\mathrm{p}$-channel silicongate metal-oxide-semiconductor (MOS) technology [2]. Since the circuit was designed with applications in computer peripherals in mind, an MOS clock rate of $2 \mathrm{MHz}$ was adequate and MOS technology allowed a high packing density in combination with high yield. n-MOS processing can be used with the same design, with approximately $2 \frac{1}{2} \times$ performance advantage.

The multicomparator utilizes a 3-phase static-dynamic shift register cell [Fig. 3(a)]. The static feature is attained by adding a clocked feedback transistor to a standard 2-phase dynamic shift register cell. The clocking diagram is shown in Fig. 3(b). In dynamic operation, $\phi_{1}$ and $\phi_{2}$ are clocked sequentially while $\phi_{3}$ is kept low. $\phi_{3}$ serves as the clamping clock which holds data in the shift register. During static operation, $\phi_{1}$ is low (isolating each cell of the shift register) and both $\phi_{2}$ and $\phi_{3}$ are high. $\phi_{3}$ gates the output of an element back to the input, and hence during the transition between modes of operation, $\phi_{3}$ must be delayed somewhat from $\phi_{2}$ to avoid an undefined data state. In practice, one may use a delayed $\phi_{2}$ as $\phi_{3}$ for both static and dynamic operation.

A unique five device activated EXCLUSIVE-NOR gate was implemented to perform the compare operation [Fig. 4(a)]. The cross-coupled structure was chosen over the standard EXCLUSIVE-NOR circuit for layout simplicity. However, the operation is similar to an EXCLUSIVE-NOR gate with the addition of an activating transistor [Fig. 4(b)]. When the activating transistor is on, the comparator is operational. When off, the comparator is disabled and the compare output indicates a match irrespective of comparator inputs. Note that the match state is a logical ONE, hence when several ExCLUSIVENOR gates are connected in parallel to construct a comparator, only one not-compare element is necessary to pull the compare line down to the low state.

The maximum operating speed of the comparator is set by the compare line capacitance and its associated pickup device resistance. A single not-compare bit must be able to pull

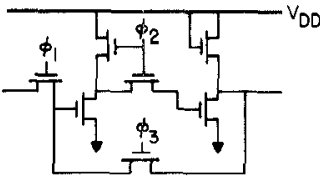

(a)

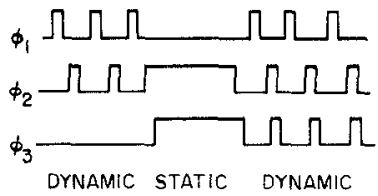

(b)
Fig. 3. Basic shift register cell. (a) Schematic. (b) Clock timing.

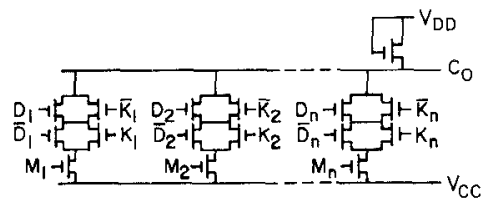

(a)

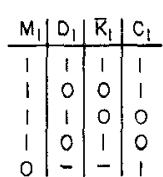

(b)
Fig. 4. Gated exclusive-Nor gate. (a) Schematic. (b) Truth table.

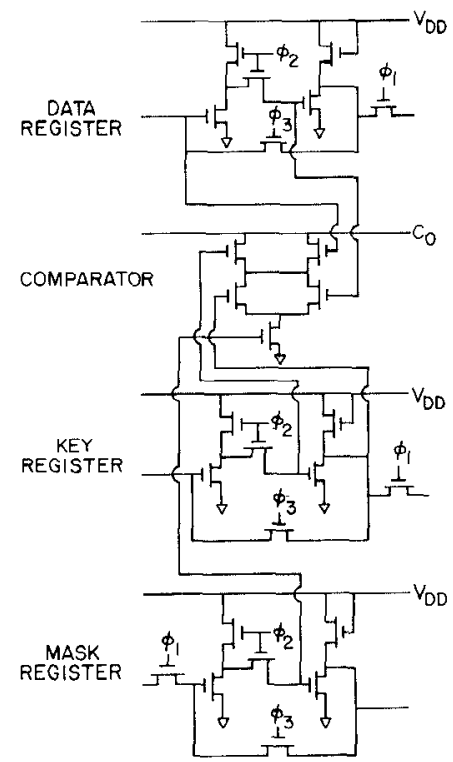

Fig. 5. Full schematic of one bit slice of the multicomparator.

the compare line down in one clock cycle. With the comparator shown, the compare line capacitance for one row of 32 cells is approximately $2 \mathrm{pF}$ and the oN resistance of the 3 series comparator transistors is about $6 \mathrm{k} \Omega$. Hence the time constant of a low going not-compare output from one row is approximately 12 ns. However, for a high going (compare) signal, the time constant is set by the $60 \mathrm{k} \Omega$ pull-up resistor at the end of the compare line. Thus the high going time constant is much larger ( $\sim 120 \mathrm{~ns})$. For this reason, the chip was arranged in four independent rows of 32 bits each. The four compare lines were NANDed to avoid paralleling the line capacitances and hence maintain the data rate achievable with the basic 32-bit row.

The schematic of one bit of the multicomparator is shown in Fig. 5. The circuit is composed of three static-dynamic shift registers for data handling and an EXCLUSIVE-NOR gate to accomplish the compare function. The unit cell was laid out using conservative design rules $(10-\mu$ metal lines with $10 \mu$ 


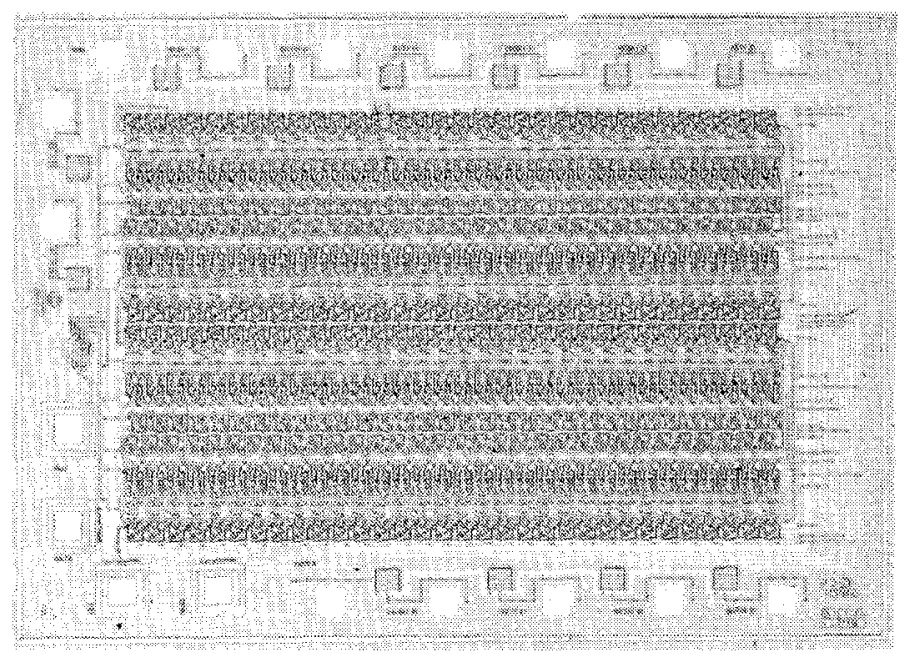

Fig. 6. Photomicrograph of multicomparator chip.

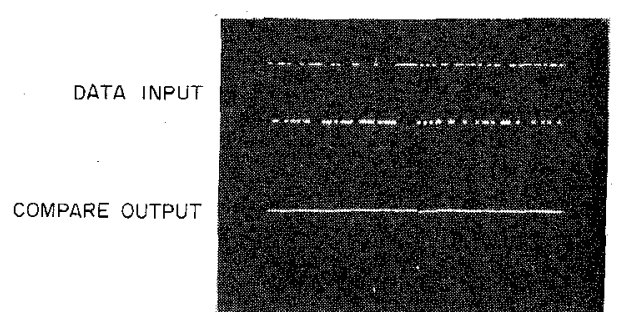

Fig. 7. Oscilloscope photo of multicomparator output with pseudorandom data input. Note: Compare pulse when data are aligned with internal stored-bit pattern.

metal-to-metal spacing). On silicon, the unit cell measures $91 \times 470 \mu$. Photomicrographs of the 128-bit multicomparator unit cell and chip layout are shown in Fig. 6. Each shift register has independent clocks and separate output pads. The 3350 device multicomparator is $107 \times 150$ mils and has 18 bonding pads.

\section{Performance Characteristics}

A typical device characterization was carried out by entering a 128-bit data word composed of a pseudorandom sequence of ones and zeros in the key register. The comparator was enabled by simultaneously entering zeros in the mask register. The 128-bit data word was then cycled through the data register. Only when alignment between the cycling data word and its stored counterpart was achieved did match occur (Fig. 7). As expected, the compare pulses had a width equal to the time increment between $\phi_{2}$ and $\phi_{1}$ and recorded every 128 even pulses. When all ones are entered in the mask register, the comparator is disabled and the compare output is high (indicating a match) since there are no unmasked bits to disagree with the data register.

The multicomparator operates over a wide range of voltages with a TTL input. Fig. 8 shows the operational range of the multicomparator at a clock frequency of $2 \mathrm{MHz}$. The clocks had a 25 percent duty cycle and were symmetrically spaced in time. $V_{c c}$ was set at $+5 \mathrm{~V}$ with $V_{\phi}$ referenced to it. The minimum supply voltage is set by two MOS thresholds (approximately

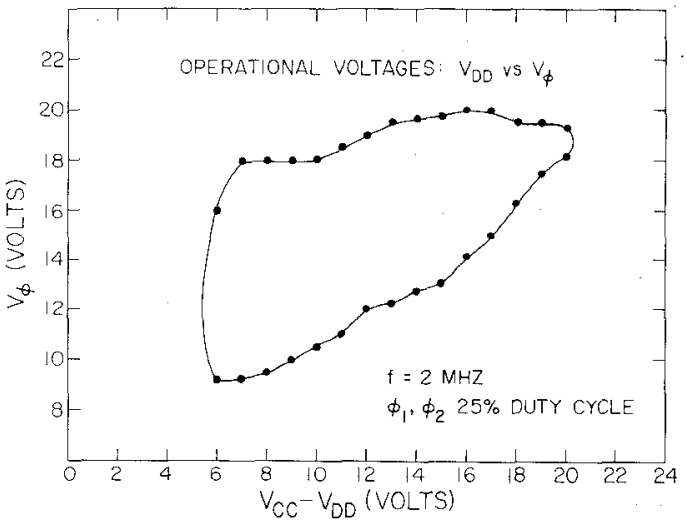

Fig. 8. Operational range of $\mathrm{p}$-channel multicomparator chip.

TABLE I

\begin{tabular}{lc}
\hline \multicolumn{1}{c}{ Parameter } & Performance $^{\mathrm{a}}$ \\
\hline Clock rate & $0.0001-2 \mathrm{MHz}$ \\
Dynamic supply current & $25 \mathrm{~mA}$ \\
Static supply current & $30 \mathrm{~mA}$ \\
Clock leakage current $\left(\phi_{1}\right)$ & $120 \mathrm{nA}$ \\
Clock leakage current $\left(\phi_{2}\right)$ & $300 \mathrm{nA}$ \\
Clock capacitance $\left(\phi_{1}\right)$ & $40 \mathrm{pF}$ \\
Clock capacitance $\left(\phi_{2}\right)$ & $60 \mathrm{pF}$ \\
Clock capacitance $\left(\phi_{3}\right)$ & $40 \mathrm{pF}$ \\
Interclock capacitance & $7 \mathrm{pF}$ \\
Input capacitance & $10 \mathrm{pF}$ \\
Output capacitance & $10 \mathrm{pF}$ \\
\hline aTest Conditions: & \\
$T=23^{\circ} \mathrm{C}, V_{c c}=5 \mathrm{~V}, V_{D D}=-5 \mathrm{~V}$, \\
$V_{\phi L}=+5 \mathrm{~V}, V_{\phi H}=-5 \mathrm{~V}, V_{\text {input }}=0.5 \mathrm{~V}$.
\end{tabular}

$2 \mathrm{~V}$ in the p-MOS process used). The maximum clock voltage was set by the onset of inversion under the field oxide. At high power supply voltages, the clock voltages were approximately one threshold less than $V_{c c}-V_{D D}$.

The multicomparator performance characteristics are summarized in Table I. 


\section{ACKNOWLEDGMENT}

The authors are pleased to acknowledge Intel Corporation for wafer fabrication.

\section{REFERENCES}

[1] T. C. Chen, "Distributed intelligence for user-oriented computing," in 1972 Fall Joint Comput. Conf., AFIPS Conf. Proc., vol. 41. Montvale, NJ: AFIPS Press, 1972, pp. 1049-1056.

[2] F. Faggin and T. Klein, "Silicon gate technology," Solid-State Electron., vol. 13, pp. 1125-1144, 1970.

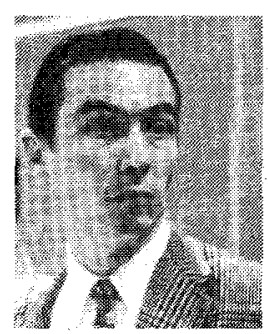

Carver A. Mead received the B.S., M.S., and Ph.D. degrees from the California Institute of Technology, Pasadena, in 1956, 1957, and 1959 , respectively.

He has been a member of the faculty of the California Institute of Technology, Pasadena, CA, since 1957. His research has contributed to the understanding of tunneling in solids, current flow mechanisms in thin dielectric films, metal-semiconductor barriers, band energies in semiconductors, and electronic processes in insulators. He has proposed and demonstrated the operation of a number of new solid-state electronic devices and holds several U.S. Patents.

Dr. Mead is a Fellow of the American Physical Society and a member of Sigma Xi.

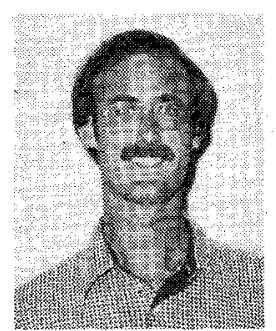

Richard D. Pashley (M'75) was born in Ft. Belvoir, VA, on September 15, 1947. He received the B.A. degree in physics from the University of Colorado, Boulder, CO, in 1969 and the M.S. and Ph.D. degrees in electrical engineering from the California Institute of Technology, Pasadena, CA, in 1970 and 1974, respectively.

In 1973 he joined the staff of Intel Corporation, Santa Clara, CA, where his work has included MOS device modeling, process development, and static RAM design. He is presently Manager of high performance MOS technology development at Intel.

Dr. Pashley is a member of Phi Beta Kappa, Sigma Xi, and Bohmische Physikalische Gesellschaft.

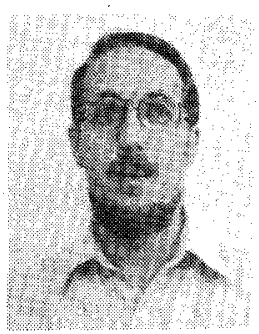

Lee D. Britton received the B.S.E.E. degree from the California Institute of Technology, Pasadena, in 1973.

He then invented and marketed a computeraided memory for theater lighting control, and now works for Hewlett-Packard Laboratories, Cupertino, CA, designing and testing LSI integrated circuits for mini-computers.

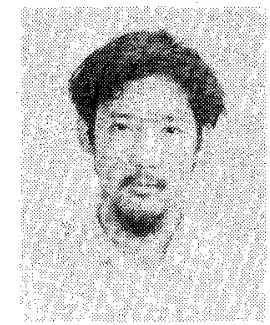

Yoshiaki T. Daimon was born in Kyoto, Japan, on July 4, 1948. He received the B.S., M.S., and Ph.D. degrees from the California Institute of Technology, Pasadena, in 1971, 1972, and 1975 , respectively.

Since 1967, he has served several research groups in the Institute. He worked as a Data Processor in hydraulics from 1967 to 1969 , engaging in the analysis of the pressure distribution of solitary waves, the influence of the geological features of a harbor upon the induced standing-wave amplitude in the harbor, and the diffusion mechanism of polluted objects in moving fluids. From 1969 to 1971, he worked as an Experimentalist in the Material Science Department and studied the switching and other electronic properties of newly developed amorphous alloys from the low temperature of $4 \mathrm{~K}$ to room temperature. From 1971 to 1975 he was a Research and Teaching Assistant both in the Electrical Engineering and Physics Departments at the California Institute of Technology. In the summer of 1971 and 1973, he visited Sony Corporation, Tokyo, Japan, as a Product-Appraisal Engineer at the Atsui plant and engaged in developments and applications of bipolar technologies in video and power integrated circuits. He is presently with the Sony Corporation, Tokyo, Japan. His interests lie in the areas of digital and linear integrated circuit designs, the physics of microelectronics, and artificial intelligence.

Stewart F. Sando, Jr. (M'73) was born in Hartford, CT, in 1949. He received the B.S. degree in physics in 1971 and the M.S.E.E. degree in 1972 from the California Institute of Technology, Pasadena. He received the M.B.A. degree from Stanford University, Stanford, CA, in 1974.

In 1973 he joined Siliconix, Inc., Santa Clara, CA, where he was employed as an Applications Engineer. In 1974 he joined Intel Corporation, Santa Clara, CA, where he is presently a Memory Components Product Marketing Engineer. 